\title{
Computing the ultrafast and radiationless electronic excited state decay of cytosine and 5-methyl-cytosine cations: uncovering the role of dynamic electron correlation
}

\author{
Dr. Javier Segarra-Martít $\quad$ Mr. Thierry $\operatorname{Tran}^{\dagger}$ \\ Prof. Michael J. Bearpark*†
}

May 23, 2019

\begin{abstract}
Photoionisation in DNA, i.e. the process of photoinduced electron removal from the chromophoric species - the nucleobases - leading to their cationic form, has been scarcely studied despite being considered to be responsible for significant damaging instances in our genetic material. In this contribution we theoretically characterise the electronic ground and excited state decay pathways of cationic DNA nucleobase cytosine ${ }^{+}$and its epigenetic derivative 5-methylcytosine $^{+}$, including the effects of dynamic electron correlation on energies and geometries of minima and conical intersections. We do this by comparing the results of XMS-CASPT2 calculations with CASSCF estimates and we find some significant differences between the results of these two methods. In particular, including dynamic electron correlation is found to significantly reduce the barrier to access the $\left(D_{1} / D_{0}\right)$ conical intersection. We find notable similarities in both cytosine and 5-methyl-cytosine cations, accessible conical intersections in the vicinity of the Franck-Condon region are found. This points towards an ultrafast depopulation of their electronic excited states. Moreover, the shape of the ground state potential energy surface strongly directs the decaying excited state population towards the cationic ground state minimum on ultrafast timescales, preventing photo-fragmentation and thus explaining their photostability. To better compare our calculations with the available experimental data we compute the UV (ground and excited state) and IR absorptions.
\end{abstract}

\footnotetext{
*corresponding author

${ }^{\dagger}$ Department of Chemistry, Molecular Sciences Research Hub, Imperial College London, White City Campus, 80 Wood Lane, W12 0BZ, London, UK; E-mail: j.segarramarti@imperial.ac.uk, m.bearpark@imperial.ac.uk
} 


\section{Introduction}

Photoinduced phenomena in DNA continues to be a topic of great interest to the scientific community due to its links with growing healthcare concerns such as skin cancer melanoma. $[1,2]$ Countless efforts have been made in recent years to understand the wide range of photo-processes triggered in our genomic material upon UV-light exposure, which span from localised excitations in individual nucleobases (monomers), [3, 4, 5, 6] delocalisation over inter-strand Watson-Crick base pairs[7] and intra-strand $\pi$-stacked motifs $[8,9,10]$ among adjacent bases (i.e. dimers) to more extended effects covering multiple bases (i.e. multimers) that increases in size and complexity. $[11,10]$

Most studies so far have focused on the effects of low-energy UV-A/B laser pulses, as these are akin to the type of incident sunlight radiation we are exposed to on a daily basis, which is not fully filtered by the ozone layer and that is believed to trigger a number of photochemically productive reactions (mutations) in our genomic material. [2, 8, 12] Less attention has been paid however to the effects of ionising radiation, which consists of higher-energy (UV-C and beyond) light sources, able to remove electrons from the chromophoric species in DNA, i.e. the nucleobases, producing their cationic forms. Cations are also known to be generated in dimeric and multimeric DNA/RNA species upon UVA/B exposure, where charge transfer states between nucleobases lead to charge separation generating cationic and anionic species.[7, 13]

The effects of high-energy radiation on DNA are and have been of interest to the scientific community as they are considered to be responsible for well-known damaging instances such as DNA cross links, base releases and single/double strand breaks occurring under ionising or Alpha-particle irradiation.[14, 15, 16] Moreover, the emergence of spectroscopic methods in the vacuum and extreme UV (VUV/EUV) and the soft and hard X-ray regimes further motivates their study, as these high-energy spectroscopic techniques allow observing photoexcitation and photoionisation processes with unprecedented accuracy[17, 18, $15]$ and enable monitoring the electronic and nuclear dynamics separately for the first time.[17, 19, 20]

While cationic species are involved in the above-mentioned DNA damaging instances, very little is known about their decay mechanisms: either theoretically or experimentally. Not much is known either regarding their formation and subsequent relaxation, which is believed to lead to photo-fragmentations, the lifetimes of which remain uncertain.

In this article we aim to explore the ultrafast radiationless decay of excited state cationic cytosine and 5-methyl-cytosine with dynamically correlated electronic structure methods, and to provide spectral fingerprints that may allow monitoring these photo-processes experimentally in the near future. We have already studied the other pyrimidine bases;[21] our purpose here is to understand what their similarities and differences are.

The ionisation of cytosine derivatives has been a topic of scarce study in the literature, with the only references found by us being relative to the specific UV/Vis absorption spectral fingerprints of cytosine ${ }^{+}[22]$ as well as their 
IR features.[23] Very little is also known about the cationic excited state decay relaxation, particularly comparing to other similar systems like the RNA nucleobase uracil.[24, 25, 26, 27, 21] The only works available in the literature are characterisation of the Dyson norms of cytosine[28] and the evolution of the cationic manifold along the relaxation of the bright and initially accessed singlet $\pi \pi^{*}$ decay channel by Matsika and co-workers, [29, 30] which cover different facets of potential ionisation mechanisms while not depicting the actual cation generation and its excited state decay relaxation channels.

Here, we characterise the ground and excited state critical structures (i.e. minima and conical intersections connecting them) that depict the excited state decay of cationic cytosine and 5-methyl-cytosine using state-of-the-art XMSCASPT2 energy gradients and couplings for the first time. We compare these results with cost-effective CASSCF estimates. [31, 32, 33, 34] By doing this we find that dynamic electron correlation mainly affects the potential energy barrier connecting the $D_{1}$ minimum with the $\left(D_{1} / D_{0}\right)_{C I}$ that controls the decay to the cationic ground state, which is a barrierless path at XMS-CASPT2 while presenting a sizeable energy barrier at CASSCF.

Ground and excited state absorptions computed on top of the characterised $D_{0}$ and $D_{1}$ minima display similar signals in the high-energy UV-B/C region while showing a well-defined fingerprint in the UV-A/Vis window related to the $D_{0}$ state. IR signals appear to be more significantly affected by being blueshifted upon 5-methylation by going from cytosine to 5-methyl-cytosine, both showing coupled carbonyl stretching and $\mathrm{NH}_{2}$ scissoring bands at $\sim 1700 \mathrm{~cm}^{-1}$, which appears within probe windows currently employed experimentally and that could be used to monitor the photoionisation dynamics.

\section{Results and Discussion}

\section{Cytosine $^{+}$}

\section{Vertical excitation energies}

We start by considering the ionisation potentials (both vertical and adiabatic) of cytosine $^{+}$, analysing the results obtained and comparing them to the available computational and experimental evidence in the literature.

By averaging the values for each electronic cationic state within the different formulations of the CASPT2 method (see Table 1) we predict the first four vertical ionisation potentials relative to the ${ }^{2} \pi_{H O M O}^{+},{ }^{2} n_{O}^{+},{ }^{2} \pi_{H O M O-1}^{+}$and ${ }^{2} n_{N}^{+}$ states (see Figure 1) to be placed at 8.74, 9.44, 9.59 and $9.94 \mathrm{eV}$, respectively, while the first adiabatic ionisation potential is situated at $8.56 \mathrm{eV}$, which is in good agreement with the available experimental evidence[35, 38, 39, 36] and previous theoretical estimates. [40, 41] A standard deviation of $\sim 0.2 \mathrm{eV}$ for the CASPT2 average is obtained for all states. Whilst $D_{0}$ and $D_{1}$ vertical ionisation and $D_{0}$ adiabatic CASPT2 averaged values fall within the registered experimental data, larger differences of $\sim 0.3$ and $\sim 0.4 \mathrm{eV}$ are found for $D_{2}$ and $D_{3}$, respectively. Similarly to what we have found in recent works on cationic 


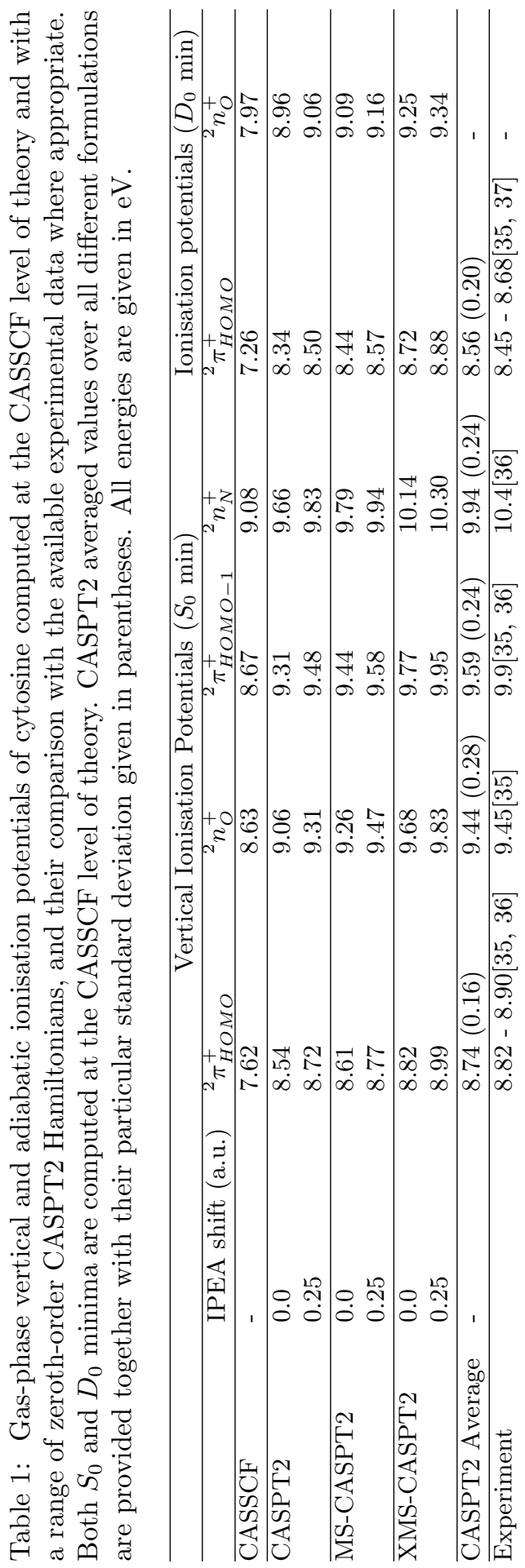



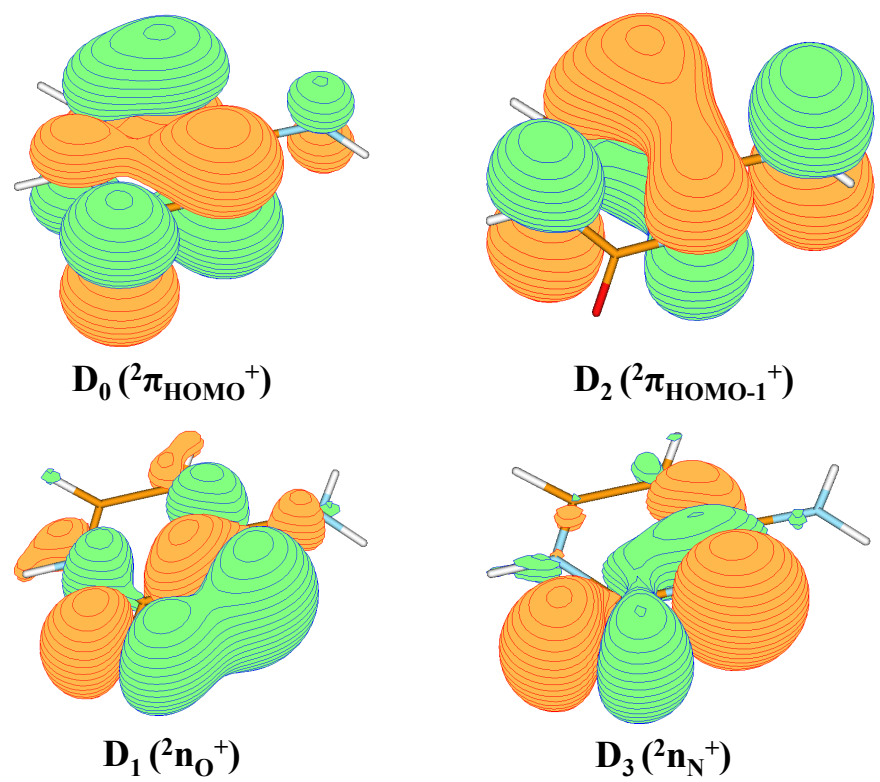

Figure 1: The singly occupied molecular orbitals characterising the four lowestlying electronic states of the cytosine cation studied here. Singly occupied molecular orbitals for 5-methyl-cytosine are omitted as they are analogous as those shown here. The different states have been labelled according to their adiabatic energy ordering (i.e. $D_{3}>D_{2}>D_{1}>D_{0}$ ), having the nature of the state in the reference $\mathrm{FC}$ region in brackets. 
uracil and thymine,[21] the use of the IPEA shift blue-shifts the estimates by $\sim 0.2 \mathrm{eV}$ and overestimates the experimental data when used in conjunction with multistate CASPT2 variants (either MS or XMS), as these already feature ionisation energies closer to the experimental range within IPEA $=0.0$ a.u.

CASSCF places the lowest-lying four vertical and first adiabatic ionisation potentials at $7.62,8.63,8.67,9.08$ and $7.26 \mathrm{eV}$, respectively (Table 1), which deviate from the experimental evidence by over $1 \mathrm{eV}$ in most cases. Despite these disagreements, CASSCF still provides acceptable energy levels when considering solely the cationic manifold and the differences between cationic states: the $D_{0}-D_{1}$ energy gap is $1.02, D_{1}-D_{2}$ is 0.04 , and $D_{2}-D_{3}$ is $0.41 \mathrm{eV}$, which are comparable to the averaged CASPT2 values for the same energy differences of $0.69,0.15$ and $0.36 \mathrm{eV}$, respectively, while preserving the correct ordering of the states.

The largest deviation between CASSCF and CASPT2 is registered for the $D_{0}-D_{1}$ energy gap, where a $\sim 0.3 \mathrm{eV}$ difference is encountered, while providing values within a tenth of an $\mathrm{eV}$ for the other two energy differences. Albeit significant, these differences are still smaller than those normally observed in the singlet manifold and do not alter the electronic excited state ordering, thus supporting the use of CASSCF as a cost-effective option to map the photoionisation events in cytosine.

\section{Geometries}

We look next at the distortions exerted in the geometries of the cationic manifold upon photoionisation with respect to the starting singlet FC $\left(S_{0}\right)$ equilibrium geometry with the critical structures optimised with the XMS-CASPT2 level of theory (Fig. 2, distances in red).

The $D_{0}\left({ }^{2} \pi_{\text {HOMO }}^{+}\right)$minimum shows C5-C6 bond elongation of $0.03 \AA$ with respect to $S_{0}$, which reflects the singly occupied molecular orbital characterising this cationic state (see Fig. 1). The $D_{1}\left({ }^{2} n_{O}^{+}\right)$minimum, on the other hand, shows significant distortions around the $\mathrm{C} 2-\mathrm{O}$ carbonyl and surrounding atoms where the cationic state localises (see Fig. 1), displaying a lengthening for $\mathrm{C} 2-\mathrm{O}$ of $0.09 \AA$, and a shortening of 0.07 and $0.08 \AA$ for the $\mathrm{N} 1-\mathrm{C} 2$ and $\mathrm{C} 2-\mathrm{N} 3$ bonds, respectively.

The $D_{3} / D_{2} \mathrm{CI}$ is situated close to the $\mathrm{FC}$ region and thus presents very small distortions, the largest being a lengthening of $0.02 \AA$ of the $\mathrm{C} 2-\mathrm{N} 3$ bond. A very similar situation is encountered for the $D_{2} / D_{1} \mathrm{CI}$, where small distortions are featured with respect to the starting FC geometry with a $0.02 \AA$ elongation along the N3-C4 bond as the main distortion. These small differences can be attributed to the effective 3-state degeneracy found very close to the FC region, which showcases the close proximity of the $D_{3}, D_{2}$ and $D_{1}$ cationic excited states upon ionisation. The $D_{1} / D_{0} \mathrm{CI}$, on the other hand, presents much more pronounced distortions showing a $0.09 \AA$ elongation of the $\mathrm{C} 2-\mathrm{O}$

bond and shortenings of $0.08,0.07$ and $0.03 \AA$ for the $\mathrm{N} 1-\mathrm{C} 2, \mathrm{C} 2-\mathrm{N} 3$ and C6-N1, respectively.

We analyse next the effect of including dynamic electron correlation on ge- 


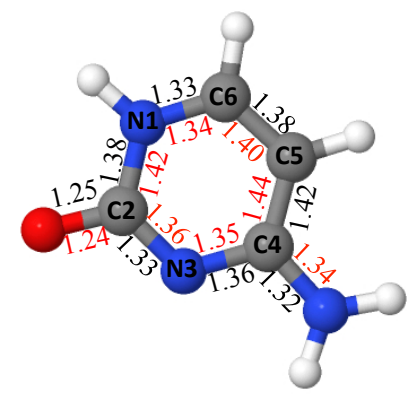

$\mathrm{D}_{0}\left({ }^{2} \pi_{\mathrm{HOMO}}{ }^{+}\right) \min$

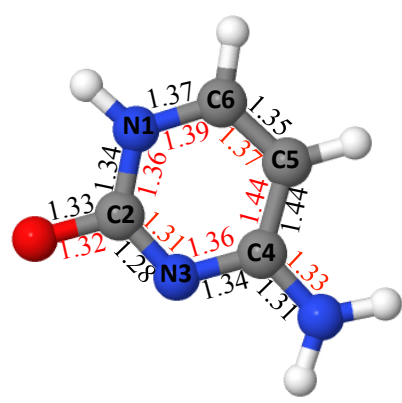

$D_{1}\left({ }^{2} \mathbf{n}_{0}^{+}\right) \min$
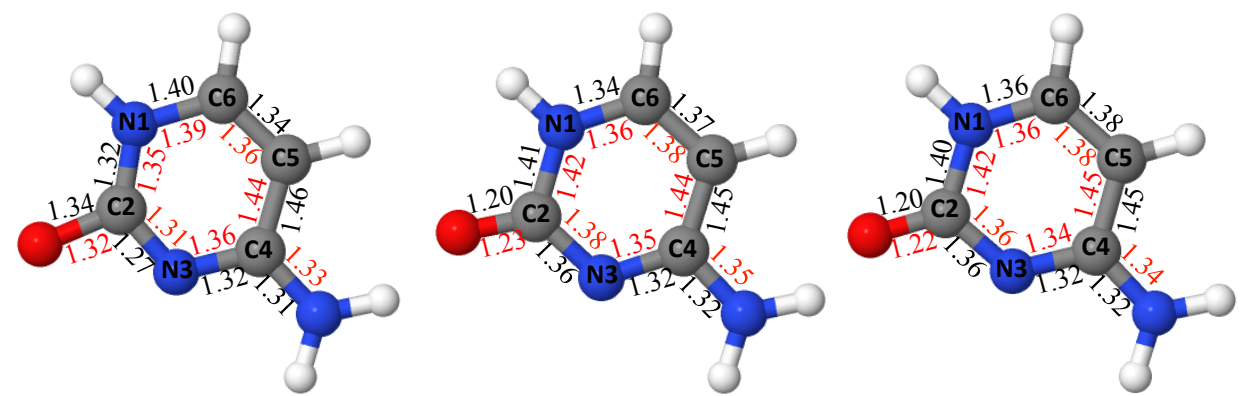

$\mathbf{D}_{1}\left({ }^{2} \mathbf{n}_{\mathrm{O}}{ }^{+}\right) / \mathbf{D}_{0}\left({ }^{2} \pi_{\text {номо }}+\right) \mathbf{C I}$

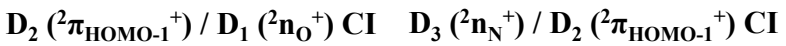

Figure 2: Minima (min) and conical intersections (CI) characterised for the lowest-lying cationic states of cytosine. All bond lengths are given in $\AA$. CASSCF values in black; CASPT2 values in red. 
ometry changes, by comparing the XMS-CASPT2 estimates mentioned above with optimised structures obtained at the CASSCF level (Fig. 2, distances in black). Noticeable differences are observed for the $D_{0}$ minimum in the N1-C2 and $\mathrm{C} 2-\mathrm{N} 3$ bonds, showing shorter bonds by 0.04 and $0.03 \AA$ respectively compared to XMS-CASPT2. The $D_{1}$ minimum features small bond shortenings of 0.02-0.03 $\AA$ across the whole structure when comparing CASSCF against the XMS-CASPT2 reference.

For the $D_{3} / D_{2}$ and $D_{2} / D_{1}$ CIs, we also find that the differences between CASSCF and XMS-CASPT2 are very small, with overall bond shortenings of 0.02-0.03 $\AA$. The $D_{1} / D_{0}$ crossing, on the other hand, shows larger differences, with bond shortenings of 0.04 for $\mathrm{N} 1-\mathrm{C} 2$ and $\mathrm{C} 2-\mathrm{N} 3$, respectively.

Overall, CASSCF and XMS-CASPT2 geometries are shown to be qualitatively similar for all different key structures characterised, which potentially validates the use of CASSCF as a potential cost-effective alternative to simulate photoionisations in cytosine. It is also worth noting that the differences induced due to the inclusion of dynamic electron correlation are much smaller than those observed in closely related systems such as uracil and thymine,[21] where XMS-CASPT2 geometries were shown to deviate more prominently from CASSCF estimates, particularly at conical intersections.

\section{Evolution and decay}

Fig. 3 displays the photochemical pathway followed by cytosine upon photoionisation of the highest-lying $D_{3}\left({ }^{2} n_{N}^{+}\right)$state down to its deactivation to the cationic ground state $\left(D_{0}\right)_{\min }$. We have analysed these decay pathways employing two different computational strategies by means of (a) CASSCF and (b) XMS-CASPT2 levels of theory.

We have assumed direct population into the $D_{3}\left({ }^{2} n_{N}^{+}\right)$state as it allows us to explore all cationic states lower in energy. Moreover, an appealing aspect of photoionisation is that selection rules do not apply and thus all sorts of ionisations are accessible and can be tuned by the laser pulse employed, hence this particular initial condition chosen being also plausible and relevant experimentally.

Fig. 3b shows that initial $D_{3}\left({ }^{2} n_{N}^{+}\right)$population leads to ultrafast relaxation to the $D_{3}\left({ }^{2} n_{N}^{+}\right) / D_{2}\left({ }^{2} \pi_{H O M O-1}^{+}\right)$conical intersection. At this geometry, the $D_{1}\left({ }^{2} n_{O}^{+}\right)$state is very close in energy at the XMS-CASPT2 level. Here we first expect $D_{3} \rightarrow D_{2}$ population transfer to take place, leading directly to the $\left(D_{2} / D_{1}\right)_{C I}$, as no well-defined minimum was found for this state either.

Moreover, this conical intersection features a close-lying $D_{1}\left({ }^{2} n_{O}^{+}\right)$state that converts it into an effective 3-state intersection at the XMS-CASPT2 level, which is expected to enable also a direct $D_{3}\left({ }^{2} n_{N}^{+}\right) \rightarrow D_{1}\left({ }^{2} n_{O}^{+}\right)$decay route. Further ultrafast relaxation is expected via crossing the $D_{2} / D_{1}$ conical intersection, bringing the population swiftly down to the $D_{1}\left({ }^{2} n_{O}^{+}\right)$state, which leads barrierlessly to the $D_{1} / D_{0}$ conical intersection and to the cationic ground state.

In Fig. 3a, CASSCF results are shown to deviate from XMS-CASPT2 as follows. A small energy gap can be observed at the CASSCF level at the effective 


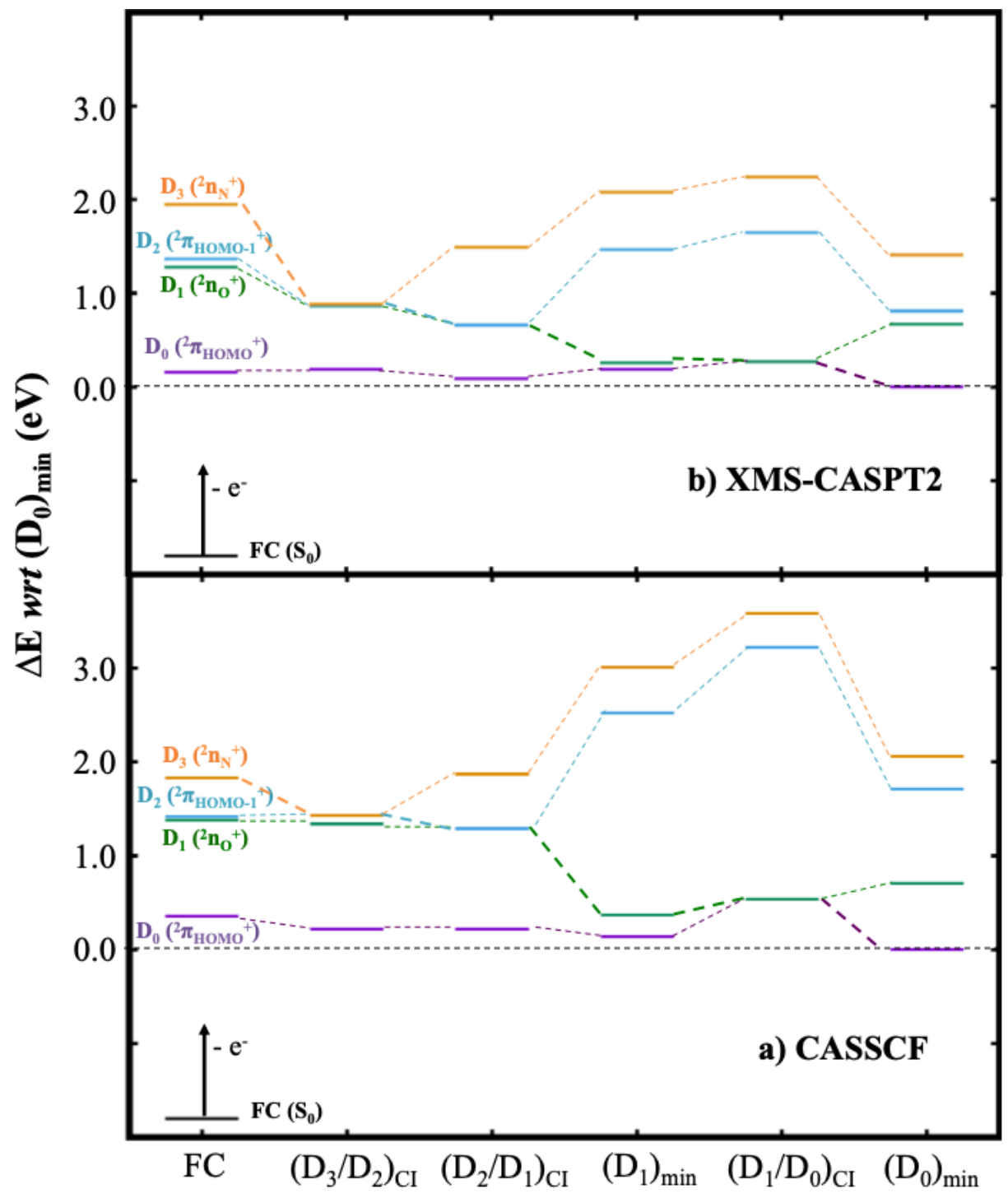

Figure 3: Potential energy surfaces of cationic cytosine computed at the (a) CASSCF and (b) XMS-CASPT2 levels of theory. All energies are given in eV with respect to $\left(D_{0}\right)_{\min }$. Thick lines represent the evolution of the excited state population assuming initial activation of the $D_{3}$ state. 
3 -state degeneracy, displaying a well-separated $D_{1}$ state at the $\left(D_{3} / D_{2}\right)_{C I}$. Dynamic electron correlation therefore has the opposite effect in cytosine from that previously reported in uracil,[21] helping to bring the electronic states together and effectively promoting a 3-state degeneracy instead of splitting the energy levels and disrupting this crossing. Significant differences are also observed when comparing the energy of the $D_{3}\left({ }^{2} n_{N}^{+}\right)$state at the FC and $\left(D_{3} / D_{2}\right)_{C I}$ geometries, showing a $0.40 \mathrm{eV}$ stabilisation at CASSCF and a more pronounced $1.06 \mathrm{eV}$ at the XMS-CASPT2 level, which appears to be correlated to the overall bond length shortenings observed with the latter level of theory. This vast energy difference points towards a more significant relaxation at the XMS-CASPT2 level along the $\mathrm{D}_{3}$ cationic state prior to accessing the CI, which dynamically translates into larger kinetic energies when reaching the crossing.

The most significant difference mechanistically between CASSCF and XMSCASPT2 is displayed at $\left(D_{1}\right)_{\text {min }}$, which features a barrierless path towards the $D_{1} / D_{0}$ intersection with XMS-CASPT2 while presenting a sizeable $\sim 0.2 \mathrm{eV}$ barrier at CASSCF.

From this we conclude that dynamic electron correlation may impact quantitatively the photoionisation process, as: i) it strongly modulates the energy difference of the $D_{3}$ state between the initially accessed FC and $\left(D_{3} / D_{2}\right)_{C I}$ geometries, featuring much larger differences at the XMS-CASPT2 level that lead to larger kinetic energy upon reaching the intersection, and ii) XMS-CASPT2 displays a barrierless decay profile for $D_{1}$ state decay, as opposed to the sizeable energy barrier characterised at the CASSCF level. Altogether, XMS-CASPT2 is expected to lead to faster $D_{1}$ decays and also to enable direct $D_{3} \rightarrow D_{1}$ population transfer through the effective 3 -state degeneracy placed in the vicinity of the FC region. Nevertheless, despite the aforementioned differences, CASSCF is still predicted to qualitatively describe the photoionisation process, the largest difference expected being an overestimated $D_{1}$ lifetime.

\section{5-methyl-cytosine ${ }^{+}$}

\section{Vertical excitation energies}

Table 2 compiles the ionisation energies obtained for 5-methyl-cytosine with CASSCF and the different CASPT2 formulations. A lesser amount of experimental data is available for this system, for which we could only find the first ionisation potential in the literature.

CASPT2 averaged values (see Table 2) predict the first four vertical ionisation potentials to be placed at $8.62,9.49,9.61$ and $9.90 \mathrm{eV}$, and the first adiabatic ionisation potential is situated at $8.30 \mathrm{eV}$. A standard deviation of $\sim 0.2 \mathrm{eV}$ for the CASPT2 average is obtained for all systems, the error associated to the choice of zeroth-order Hamiltonian being the same for the different states despite their different character.

The only experimental reference found by us for the ionisation of 5-methylcytosine places the first ionisation potential relative to the $D_{0}\left({ }^{2} \pi_{\mathrm{HOMO}}^{+}\right)$state at $8.78 \mathrm{eV}$, within $0.16 \mathrm{eV}$ of our averaged estimates.[42] This value, however, is 


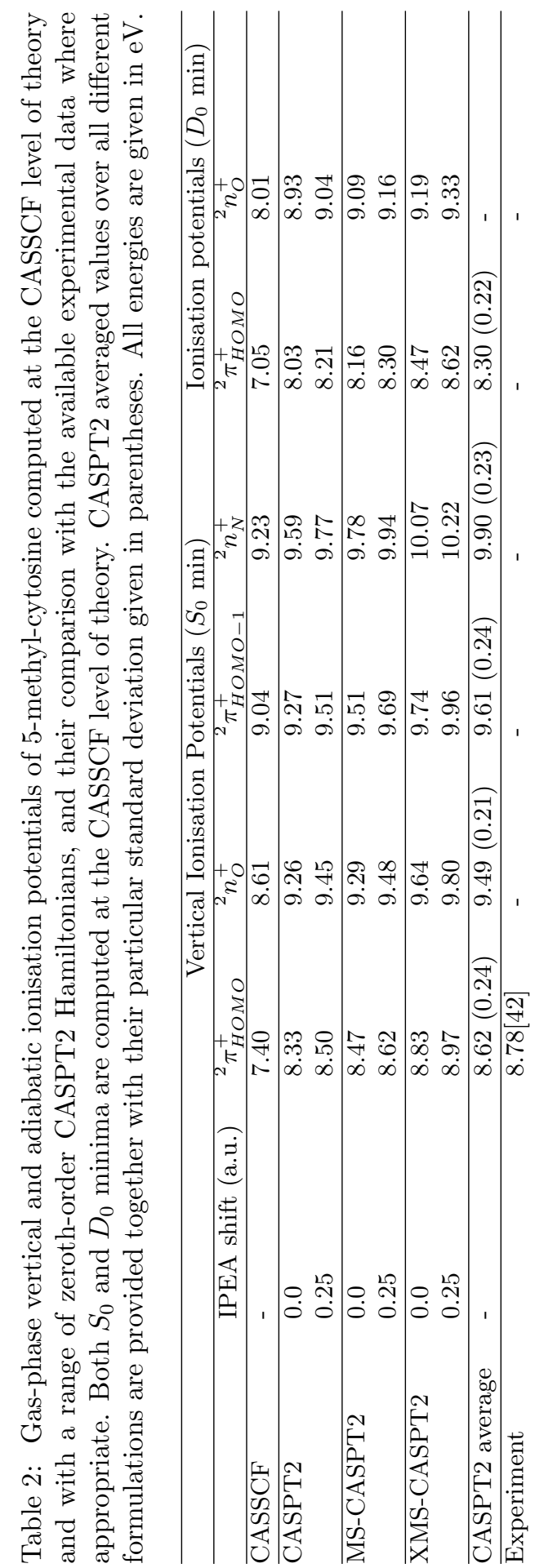


very close to those previously reported for cytosine, and suggests 5-methylation plays a lesser role in the energetics of the cationic manifold here as compared to what we recently observed for uracil and thymine.[21] This is also supported by our computations, which predict vertical ionisation potentials of 5-methylcytosine to be in line with those of cytosine, showing a red-shift of the first vertical ionisation potential of $0.12 \mathrm{eV}$ and below a tenth of an $\mathrm{eV}$ for the others, while displaying a larger $0.27 \mathrm{eV}$ red-shift for the adiabatic ionisation potential, which hints at more pronounced differences when geometry relaxation is considered.

CASSCF estimates place the first four vertical ionisation potentials at 7.40, 8.61, 9.04 and $9.23 \mathrm{eV}$, and the first adiabatic potential at $7.05 \mathrm{eV}$, all of them being roughly about an $\mathrm{eV}$ red-shifted compared to their CASPT2 counterparts and the available experimental evidence. As in cytosine, CASSCF still provides acceptable estimates when considering energy differences within the cationic manifold: the $D_{0}-D_{1}$ energy gap being $0.96, D_{1}-D_{2}$ is 0.03 and $D_{2}-D_{3}$ is $0.02 \mathrm{eV}$, which are comparable to the averaged CASPT2 values of $0.87,0.13$ and $0.28 \mathrm{eV}$, respectively, most importantly preserving the correct ordering of the different electronic states.

The largest deviation between CASSCF and CASPT2 in this case is registered for the $D_{2}-D_{3}$ energy gap, which features a $\sim 0.25 \mathrm{eV}$ difference while providing values within a tenth of an $\mathrm{eV}$ for the other cases. These differences are not too significant and, more importantly, do not modify the electronic excited state ordering.

\section{Geometries}

We analyse next the geometrical distortions with respect to the starting singlet FC equilibrium region with critical structures being optimised at the XMSCASPT2 level of theory (Fig. 4, distances in red). The $D_{0}\left({ }^{2} \pi_{H O M O}^{+}\right)$minimum shows bond elongations of $0.02,0.02$ and $0.04 \AA$ for $\mathrm{N} 1-\mathrm{C} 2, \mathrm{~N} 3-\mathrm{C} 4$ and $\mathrm{C} 5-\mathrm{C} 6$, respectively, and bond shortenings of $0.03,0.04$ and $0.02 \AA$ for $\mathrm{C} 2-\mathrm{N} 3, \mathrm{C} 4-\mathrm{N}$ and $\mathrm{C} 6-\mathrm{N} 1$, featuring larger distortions than those reported for cytosine and that extend beyond the C5-C6 moiety. The $D_{1}\left({ }^{2} n_{O}^{+}\right)$minimum, on the other hand, displays more significant distortions, featuring bond elongations of 0.10 , 0.04 and $0.03 \AA$ for $\mathrm{C} 2-\mathrm{O}, \mathrm{N} 3-\mathrm{C} 4$ and $\mathrm{C} 6-\mathrm{N} 1$, respectively, and shortenings of $0.06,0.08$ and $0.04 \AA$ for $\mathrm{N} 1-\mathrm{C} 2, \mathrm{C} 2-\mathrm{N} 3$ and $\mathrm{C} 4-\mathrm{N}$. These differences are again more pronounced than those previously found for cytosine while still presenting the carbonyl stretching as the main distortion present in this excited state minimum.

As in cytosine, the $\mathrm{D}_{3} / \mathrm{D}_{2}$ CI is situated in the vicinity of the $\mathrm{FC}$ region, the largest deviation with respect to $\left(S_{0}\right)_{\min }$ being a $0.03 \AA \mathrm{C} 2-\mathrm{N} 3$ bond shortening. Small distortions are also found for the $D_{2} / D_{1} \mathrm{CI}$, where the main deviation with respect to the $\mathrm{FC}$ is a $0.03 \AA$ elongation of the $\mathrm{N} 3-\mathrm{C} 4$ bond. Both conical intersections feature small differences with the FC region, which are more pronounced than those reported for cytosine and that lead to well-separated energy levels that feature no effective 3 -state crossings. The $D_{1} / D_{0}$ CI presents more 

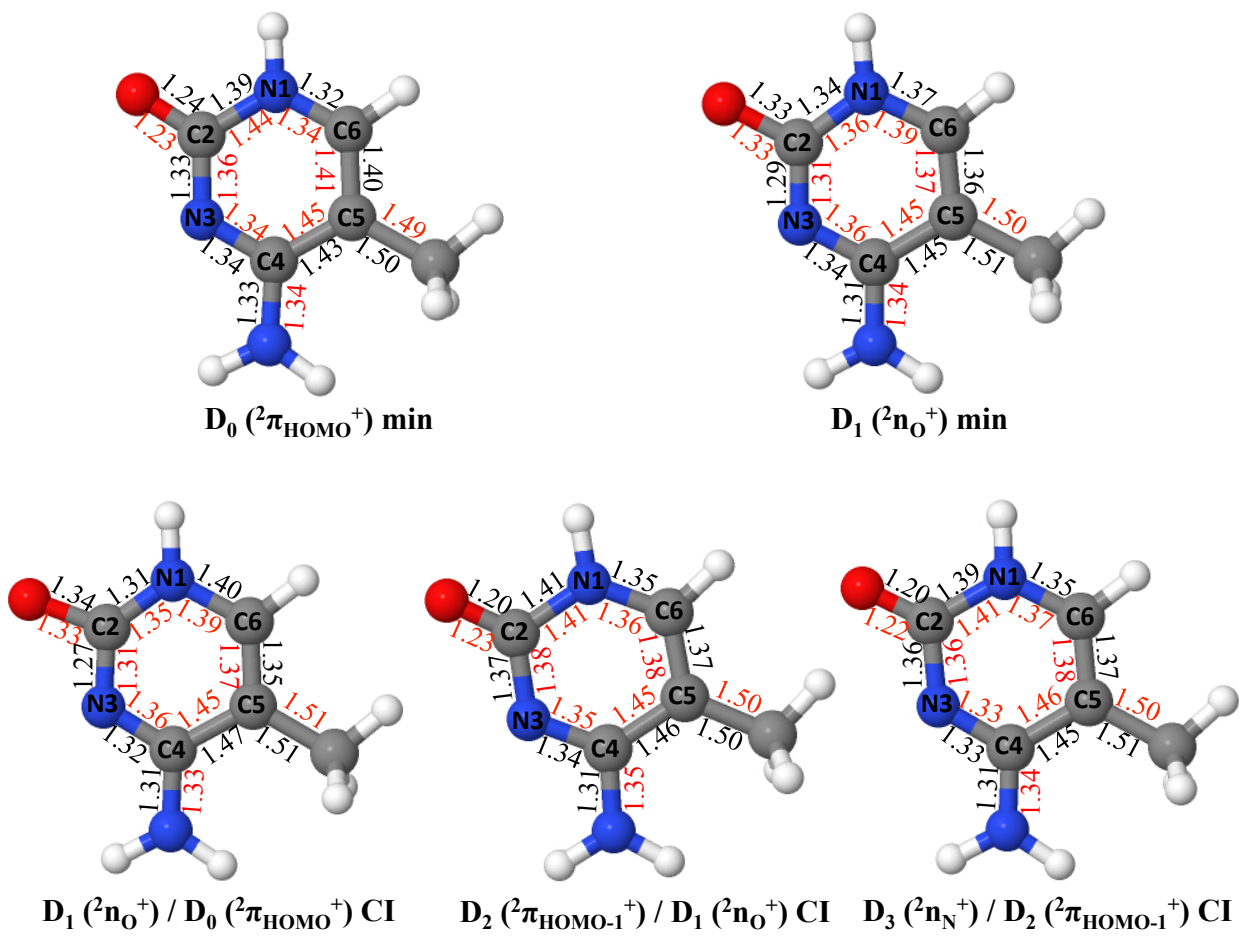

Figure 4: Minima (min) and conical intersections (CI) characterised for the lowest-lying cationic states of 5-methyl-cytosine. All bond lengths are given in A. CASSCF values in black; CASPT2 values in red. 
significant distortions showing a 0.10 and $0.03 \AA$ elongation of the $\mathrm{C} 2-\mathrm{O}$ and $\mathrm{C} 6-\mathrm{N} 1$ bonds and shortenings of 0.07 and 0.08 Åfor N1-C2 and $\mathrm{C} 2-\mathrm{N} 3$.

Inclusion of dynamic electron correlation as measured by comparing the XMS-CASPT2 estimates with optimised structures obtained at the CASSCF level (Fig. 2, distances in black) leads to relatively small differences. The most pronounced deviations are encountered for the $\mathrm{D}_{0}$ minimum along the $\mathrm{N} 1-\mathrm{C} 2$ and $\mathrm{C} 2-\mathrm{N} 3$ bonds, showing shorter bonds by 0.05 and $0.03 \AA$, respectively, compared to XMS-CASPT2. $\left(D_{1}\right)_{\text {min }}$ displays small bond shortenings of $0.02 \AA$ across the whole structure when comparing CASSCF against the XMS-CASPT2 reference as was also found for cytosine.

$D_{3} / D_{2}$ and $D_{2} / D_{1}$ CIs display similar differences between CASSCF and XMS-CASPT2 to those previously reported at the $\mathrm{D}_{1}$ minimum, featuring overall bond shortenings of $\sim 0.02 \AA$, while being dissimilar with one another and thus not presenting the effective 3-state degeneracy observed for cytosine. $D_{1} / D_{0} \mathrm{CI}$, on the other hand, is found to display the largest differences between CASSCF and XMS-CASPT2, featuring pronounced bond length shortenings of $0.04 \AA$ for $\mathrm{N} 1-\mathrm{C} 2, \mathrm{C} 2-\mathrm{N} 3$ and N3-C4.

\section{Evolution and decay}

Fig. 5 displays the photo-initiated pathways followed by 5 -methyl-cytosine upon photoionisation of $D_{3}$. As above, we have analysed the different decay channels employing two different computational strategies by means of (a) CASSCF and (b) XMS-CASPT2 levels of theory.

Fig. 5b shows that populating $D_{3}\left({ }^{2} n_{N}^{+}\right)$leads to an ultrafast relaxation to the $D_{3}\left({ }^{2} n_{N}^{+}\right) / D_{2}\left({ }^{2} \pi_{H O M O-1}^{+}\right)$conical intersection, which is responsible of funnelling down the excited state population to the $D_{2}\left({ }^{2} \pi_{H O M O-1}^{+}\right)$state. Different to cytosine, this conical intersection is well separated from the $D_{1}$ state at the XMS-CASPT2 level of theory and thus subsequent relaxation is then expected to the $\left(D_{2} / D_{1}\right)_{C I}$, leading to a sequential $D_{3}\left({ }^{2} n_{N}^{+}\right) \rightarrow D_{2}\left({ }^{2} \pi_{H O M O-1}^{+}\right)$ $\rightarrow D_{1}\left({ }^{2} n_{O}^{+}\right)$deactivation mechanism where no minima were found for either $D_{3}$ and $D_{2}$ states. Once reaching $D_{1}\left({ }^{2} n_{O}^{+}\right)$, the excited state population is expected to be trapped momentarily in the well-defined $\left(D_{1}\right)_{\min }$ which shows a negligible $0.03 \mathrm{eV}$ potential energy barrier to reach the $\left(D_{1} / D_{0}\right)_{C I}$, being thus less favourable than the barrierless decay shown above for cytosine ${ }^{+}$. Upon reaching $\left(D_{1} / D_{0}\right)_{C I}$, the population is expected to be channelled to the cationic ground state $\left(D_{0}\right)$ and the excess energy dissipated thermally.

In Fig. 5a, CASSCF results show lesser deviations compared to XMSCASPT2 reference values for 5-methyl-cytosine, where both methods predict a 2-state instead of a 3 -state intersection between the $D_{3}$ and $D_{2}$ states in the vicinity of the FC region. Qualitatively and almost quantitatively analogous results are observed in this case when comparing the energy of the $D_{3}\left({ }^{2} n_{N}^{+}\right)$state at the $\mathrm{FC}$ and $\left(D_{3} / D_{2}\right)_{C I}$ geometries, showing energy gaps of 0.62 and 0.43 $\mathrm{eV}$ at CASSCF and XMS-CASPT2 levels of theory, thus suggesting dynamic electron correlation appears to play a lesser role in 5-methyl-cytosine compared to cytosine. 


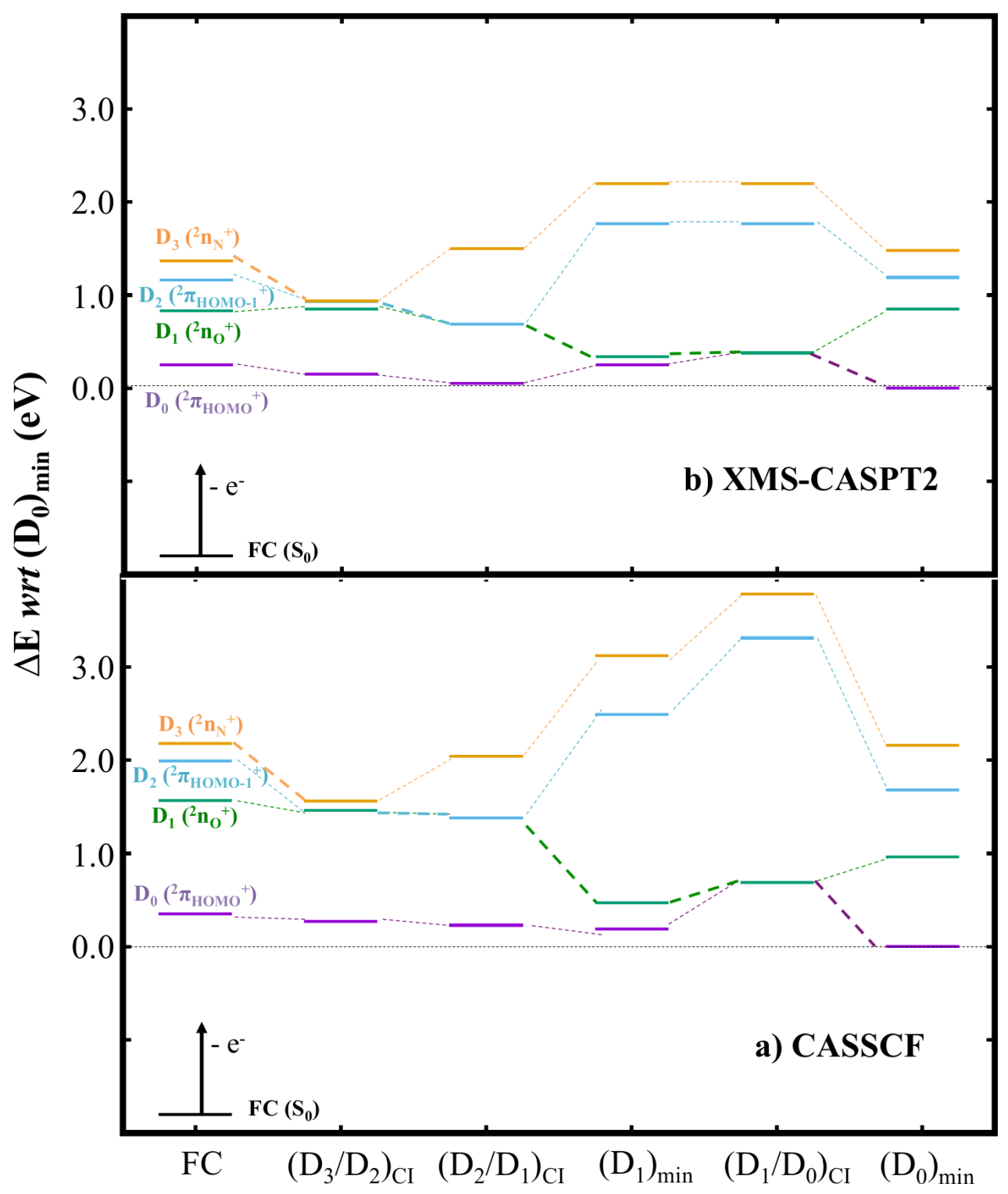

Figure 5: Potential energy surfaces of cationic 5-methyl-cytosine computed at the (a) CASSCF and (b) XMS-CASPT2 levels of theory. All energies are given in $\mathrm{eV}$ with respect to $\left(D_{0}\right)_{\min }$. Thick lines represent the evolution of the excited state population assuming initial activation of the $D_{3}$ state. 
As in cytosine ${ }^{+}$, the largest difference due to dynamic electron correlation is observed at the $\left(D_{1}\right)_{\min }$, which presents a sizeable $0.22 \mathrm{eV}$ barrier along the pathway to the $\left(D_{1} / D_{0}\right)_{C I}$, much larger than the $0.03 \mathrm{eV}$ obtained at the XMS-CASPT2 level.

We thus conclude that dynamic electron correlation in 5-methyl-cytosine has a lesser yet still noticeable effect in describing the photoionisation processes compared to cytosine, as CASSCF displays a significant potential energy barrier between $\left(D_{1}\right)_{\min }$ and $\left(D_{1} / D_{0}\right)_{C I}$ that is expected to artificially increase the lifetime of the $D_{1}$ state whilst expecting qualitatively similar results for the rest of the photo-process.

\section{Electronic and IR absorption signals}

We have next simulated the potential spectral fingerprints to monitor the photoinitiated events described above. Fig. 6 displays electronic and IR transitions originating from the two well-defined cationic ground $D_{0}\left({ }^{2} \pi_{\text {HOMO }}^{+}\right)$and excited $D_{1}\left({ }^{2} n_{O}^{+}\right)$minima.

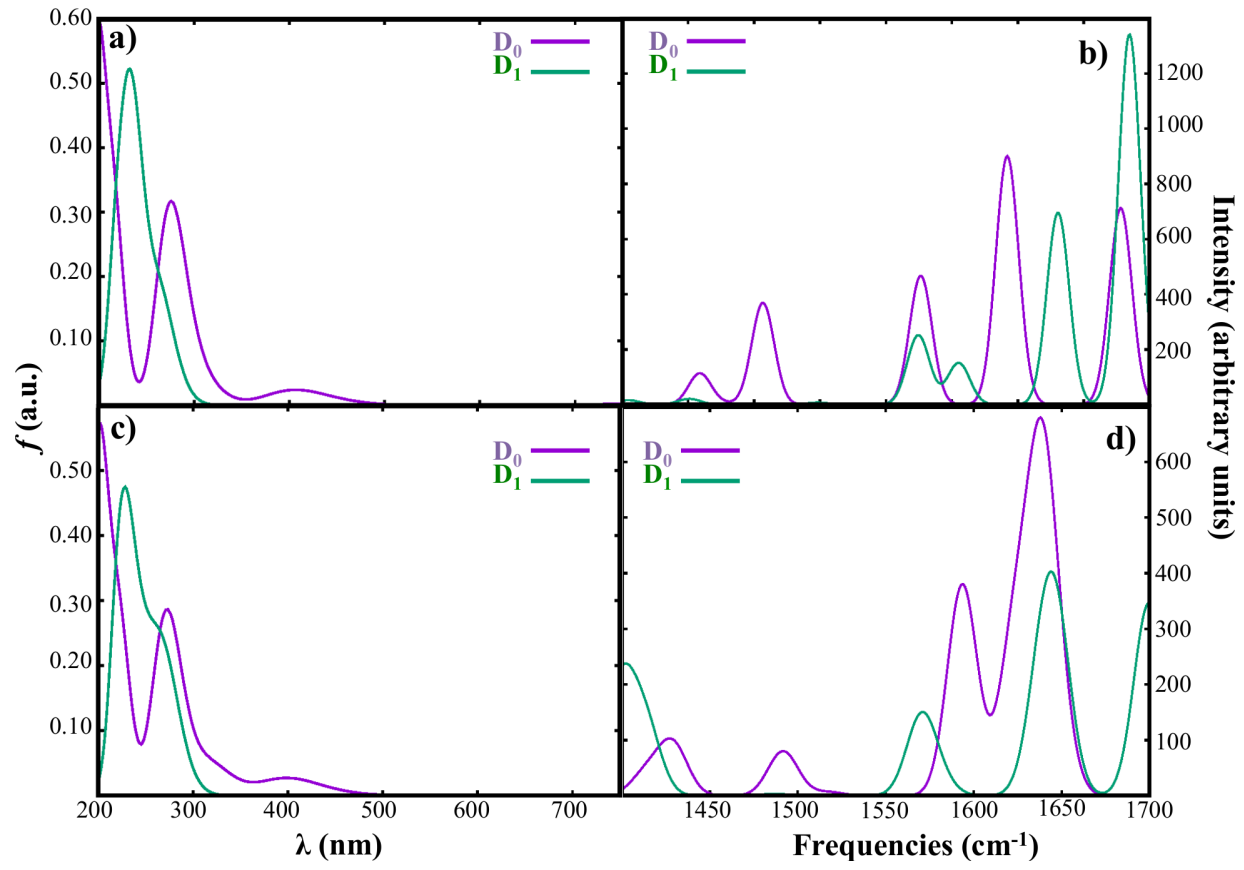

Figure 6: Electronic (excited state; panels a and c) and vibrational (IR; panels b and d) absorption signals of cationic cytosine (top panels) and 5-methyl-cytosine (bottom panels) in their ground $\left(D_{0}\right)$ and excited state $\left(D_{1}\right)$ minima.

Frequent UV/Vis probe ranges $(200-750 \mathrm{~nm})$ are considered for both ground $\left(D_{0}\right)$ and excited $\left(D_{1}\right)$ electronic state absorptions, which consists of their respective ${ }^{2} \pi^{*} \leftarrow^{2} \pi^{+}$and ${ }^{2} n_{O}^{*} \leftarrow^{2} n_{O}^{+}$dipole allowed transitions, while a 1400 - 
$1700 \mathrm{~cm}^{-1}$ probe window is shown for the IR as it is commonly reported in transient experiments.[43]

Cytosine ${ }^{+} \mathrm{UV} / \mathrm{Vis}$ and IR predicted signals are shown in the top panels (a and b) of Fig. 6. Ground and excited state absorptions in the UV/Vis window refer to those often recorded in pump-probe set-ups, which we predict will appear as an overlapping band in the high-energy UV-B/C (200-300 nm) region while showing a weak absorption band peaking $\sim 410 \mathrm{~nm}$ that is unique to $D_{0}$. IR signals also appear to be quite congested, displaying overlaps over the high-energy window $\left(1550-1700 \mathrm{~cm}^{-1}\right)$ while showing a clear peak at $\sim 1500$ $\mathrm{cm}^{-1}$ for $D_{0}$ corresponding to the C4-C5 stretching where the unpaired charge is mostly located, which agrees with the recent experimental evidence of Lesslie et al.[23] for this particular band. The $D_{1}$ state is characterised by a strong carbonyl stretching coupled to the $\mathrm{NH}_{2}$ scissoring motion also reported experimentally and placed at $\sim 1680 \mathrm{~nm},[23]$ which we find to be slightly blue-shifted with respect to the recorded evidence. It is worth noting, however, that no scaling factors are used in our spectra as we are interested in a qualitative rather than a quantitative description of the signals to recognise those that might be employed to monitor the photo-process.

Fig. 6c shows the UV/Vis spectrum for 5-methyl-cytosine ${ }^{+}$, which displays very similar signals to those described above for cytosine ${ }^{+}$while being slightly blue-shifted due to 5 -methylation. The main intense signals appear again in the high-energy (200-300 $\mathrm{nm}$ ) window and a low-energy absorption band is predicted at $400 \mathrm{~nm}$, being $\sim 10 \mathrm{~nm}$ blue-shifted with respect to the analogous transition characterised for cytosine ${ }^{+}$. The IR signals, depicted in Fig. 6d, appear to be significantly modulated by 5 -methylation by splitting the C4-C5 stretching band of $D_{0}$ at $\sim 1500 \mathrm{~cm}^{-1}$ and blue-shifting the mixed carbonyl stretching and $\mathrm{NH}_{2}$ scissoring motion beyond $1700 \mathrm{~cm}^{-1}$.

These results are consistent with the effects of 5-methylation characterised when going from uracil ${ }^{+}$to thymine ${ }^{+}$.[21] Comparing the two pairs of systems (uracil $^{+}$and thymine ${ }^{+}$vs cytosine ${ }^{+}$and 5-methyl-cytosine ${ }^{+}$) we observe a significant blue-shift in the low-energy Vis absorption signal for $D_{0}$ that is also observed for the higher lying UV signals for both $D_{0}$ and $D_{1}$. The IR spectra, on the other hand, display a well-defined C4-C5 stretching signal at $\sim 1500$ $\mathrm{cm}^{-1}$ for $D_{0}$ in cytosine ${ }^{+}$and 5-methyl-cytosine ${ }^{+}$, which is featured at both $D_{0}$ and $D_{1}$ states for both uracil ${ }^{+}$and thymine ${ }^{+}$, while featuring a blue-shifted $D_{1}$ carbonyl stretching potentially due to its coupling to the $\mathrm{NH}_{2}$ scissoring mode not featured in uracil and thymine.

Summarising, we identify the low-energy UV-A/Vis window at $\sim 400 \mathrm{~nm}$ and the low-energy $\sim 1500 \mathrm{~cm}^{-1}$ to provide the best fingerprint to monitor the dynamics of the $D_{0}$ state while the intense peak at $\sim 1700 \mathrm{~cm}^{-1}$ related to the carbonyl stretching is identified as the most prominent feature to track the $D_{1}$ cationic excited state decay. 


\section{Conclusions}

In this article we thoroughly analyse the cationic excited state decay of cytosine ${ }^{+}$ and 5-methyl-cytosine ${ }^{+}$, and characterise the role played by dynamic electron correlation by employing the XMS-CASPT2 method for the description of both energies and gradients for the first time.

We find that ionisation energies are quite sensitive to dynamic electron correlation and employ a range of CASPT2 formulations to analyse their performance with respect to the available experimental observables (vertical and adiabatic ionisation potentials). CASSCF energy differences are shown to be qualitatively correct when considering solely the cationic manifold, but the energies appear to be significantly red-shifted with respect to the available experimental evidence. 5 -methyl-cytosine barely alters energetic position of the cationic manifold with respect to cytosine, its effect being much smaller than those previously characterised for the other pyrimidine nucleobases uracil and thymine.[21]

The most notable difference observed between the two systems studied are related to the presence of an effective 3 -state $\left(D_{3} / D_{2} / D_{1}\right)$ degeneracy region present in cytosine at CASSCF and XMS-CASPT2, which is disrupted upon 5 -methylation at both levels of theory, similar to what was found in uracil and thymine. [21]

Our XMS-CASPT2 optimised potential energy surfaces show how, upon accessing initially the highest-lying $D_{3}\left({ }^{2} n_{N}^{+}\right)$state, the excited state population will reach the $\left(D_{3} / D_{2}\right)_{C I}$ and decay to $D_{2}\left({ }^{2} \pi_{H O M O-1}^{+}\right)$. The $\left(D_{3} / D_{2}\right)_{C I}$ features a close-lying $D_{1}\left({ }^{2} n_{O}^{+}\right)$state that may also facilitate direct decay to the latter via an effective 3-state degeneracy in cytosine, which is however disrupted in 5-methyl-cytosine. Excited state population is predicted to decay in an ultrafast manner from $D_{2}$ to $D_{1}$ via $\left(D_{2} / D_{1}\right)_{C I}$ and evolve along the latter state reaching a $D_{1}$ minimum we have characterised.

Inclusion of dynamic electron correlation is shown to stabilise the $\left(D_{1} / D_{0}\right)_{C I}$ on both systems, enabling an effective barrierless decay from $\left(D_{1}\right)_{\min }$ to the cationic ground state $\left(D_{0}\right)$, whereas CASSCF estimates feature a potential energy barrier along the pathway from $\left(D_{1}\right)_{\min }$ and hampering the access to $\left(D_{1} / D_{0}\right)_{C I}$, which we predict will artificially increase the lifetime of the $D_{1}$ state.

A sequential deactivation scheme is thus predicted for 5-methyl-cytosine where the excited state population follows a direct $D_{3}\left({ }^{2} n_{N}^{+}\right) \rightarrow D_{2}\left({ }^{2} \pi_{\mathrm{HOMO}-1}^{+}\right)$ $\rightarrow D_{1}\left({ }^{2} n_{O}^{+}\right) \rightarrow D_{0}\left({ }^{2} \pi_{\text {HOMO }}^{+}\right)$pathway, whereas cytosine is predicted to additionally display a significant straight $D_{3}\left({ }^{2} n_{N}^{+}\right) \rightarrow D_{1}\left({ }^{2} n_{O}^{+}\right)$decay component due to the effective 3 -state degeneracy featured in the vicinity of the FC region that may enable this route.

Computing the UV/Vis and IR ground and transient absorption signals on top of the characterised $D_{1}\left({ }^{2} n_{O}^{+}\right)$and $D_{0}\left({ }^{2} \pi_{H O M O}^{+}\right)$minima suggests 5 methylation plays a minor role in the description of the electronic spectra while showing significant effects in the IR. In the UV/Vis window, weak signals $\sim 400$ $\mathrm{nm}$ associated to the $D_{0}$ state are expected to be the most prominent finger- 
print for this state. In the IR, on the other hand, 5-methylation is shown to significantly blue-shift the signals unique to both $D_{0}$ and $D_{1}$, which are located at $\sim 1500 \mathrm{~cm}^{-1}$ for the former and $\sim 1700 \mathrm{~cm}^{-1}$ for the latter and that are based on C4-C5 and carbonyl stretching fingerprints that define those states and that could be employed to monitor the photoionisation decays of both cytosine and 5 -methyl-cytosine.

\section{Computational Details}

OpenMOLCAS[44] was used for most of the computations here reported. An atomic natural orbital basis set (ANO-L) was used throughout in its valence double- $\zeta$ polarised contraction. $[45,46]$ The active space for cytosine and 5methyl-cytosine comprises the full $\pi$ valence occupied and virtual space plus the two occupied $n$ lone pair $\left(n_{O}\right.$ and $n_{N}$ ) orbitals to include the ${ }^{2} n_{O}^{+}$and ${ }^{2} n_{N}^{+}$ states, totalling 14 electrons in 10 orbitals for the neutral and 13 electrons in 10 orbitals for the cationic species.

CASSCF wave functions were averaged over five doublet states and were subsequently used for single-point CASPT2 energy corrections.[47, 48, 49] An imaginary level shift of 0.2 a.u. was employed in the perturbative step to avoid the presence of intruder states,[50] and IPEA shifts[51] of 0.0 and 0.25 a.u. were tested as this correction has been shown to improve the description of cationic open-shell states in these systems.[40]

CASPT2 computations were performed in its single-state, [47, 48, 49] multistate (MS),[52] and extended multistate (XMS)[53] variants to benchmark the effect of the zeroth order Hamiltonian on the cationic manifold. For presenting and discussing the energies at the FC region, we have chosen to average over the different CASPT2 formulations as this allows us to show the mean value as well as the standard deviation expected by modifying the zeroth-order Hamiltonian. However, for geometries and energies away from the FC region we have only reported XMS-CASPT2 estimates as this has been shown to provide a better balance in the simultaneous description of covalent and ionic excited states[53] and therefore gives us more reliable geometries, particularly for crossing regions. $[54,55]$ It is worth noting that the IPEA shift described above was calibrated for the single-state formulation of CASPT2, 0.25 a.u. therefore being possibly a non-optimal value for correcting MS-/XMS-CASPT2 energies. We made use of this value due to its wide use in the literature, being the prescribed value for CASPT2, and to roughly estimate its effect in its multistate formulations.

Additional computations on top of the CASSCF geometry optimisations employing the SS-CASPT2//CASSCF protocol were carried out but were discarded as the system presented strong differential correlation effects leading to the appearance of artificial crossings along the decay pathways, thus discouraging its use.

The resolution of identity based on the Cholesky decomposition was used to speed up the calculation of the electron repulsion integrals, $[56,57,58]$ and was 
used for both energy evaluations[59] as well as in calculating CASSCF analytical gradients[60,61] and non-adiabatic couplings. [62] Second-order nuclear derivatives were computed numerically employing the aforementioned gradients.[63]

CASSCF conical intersections were characterised with the method of Fdez Galván et al.[62]

The characterised cationic ground $\left(D_{0}\right)$ and excited $\left(D_{1}\right)$ state minima, as well as the different low-lying conical intersections (CIs) were also optimised at the XMS-CASPT2 level of theory to establish the role of dynamic electron correlation on the geometrical parameters in these cations, as it has been shown to significantly impact the singlet manifold.[64, 65, 66, 67] XMS-CASPT2 minima and CI optimisations (using the projection method of Bearpark et al.[68]) were also carried out with analytical gradients[69, 70, 71] and couplings[72] as implemented in BAGEL[73] to ensure that those obtained using numerical differentiation are correct. XMS-CASPT2 geometry optimisations were carried out without the use of the IPEA shift, as it was shown in previous work to lead to analogous structures.[21] Only XMS-CASPT2 optimisations with analytical gradients were reported, even though analogous structures were found by computing the gradients numerically. All optimised critical points (minima and conical intersections) at both CASSCF and XMS-CASPT2 levels of theory were connected with linear interpolations in internal coordinates that confirmed the barrierless profiles connecting the reported structures.

Additional computations averaging over the lowest-lying 30 electronic doublet states were carried out on top of the different minima to evaluate the excited state absorption signals.[74] The reasoning behind 30 states was so that the CASSCF reference included the intense bright doubly excited states that feature in transient absorption (pump-probe) experiments, as it has been shown elsewhere. $[75,76]$ We have assumed that excited state absorptions of the individual ${ }^{2} n^{+}$and ${ }^{2} \pi^{+}$states are dominated by the electronic structure at their corresponding minima, $[77,78]$ thus neglecting the time-evolution of the system and its lineshape, which is costly to simulate and out of the scope of the present study. [79, 80, 81, 82] The CAS state interaction method[83] was employed to evaluate transition dipole moments and oscillator strengths and the energies were corrected with the standard (single-state) CASPT2 formulation with an IPEA shift of 0.0. The transitions were broadened with Gaussian functions with full width at half maximum of $0.3 \mathrm{eV}$, as used in similar organic systems, [84] while the IR signals were broadened with Gaussian functions with full width at half maximum of $10 \mathrm{~cm}^{-1}$.[85] Excited state absorptions and IR signals were broadened as implemented in Gabedit[86] and orbital visualisation was performed with Molden.[87]

\section{Acknowledgments}

J.S.-M. acknowledges support from the European Commission through the Marie Curie actions (AttoDNA, FP8-MSCA-IF, grant no 747662). We wish to thank Dr. João Malhado and Prof. Mike Robb for useful discussions. We also wish to 
thank the use and support of the Imperial College Research Computing Service (DOI: $10.14469 / \mathrm{hpc} / 2232$ ).

\section{Keywords}

DNA cations, 5-methyl-Cytosine, Cytosine, photoionisation, CASSCF/CASPT2, radiationless decay, photostability

\section{TOC}

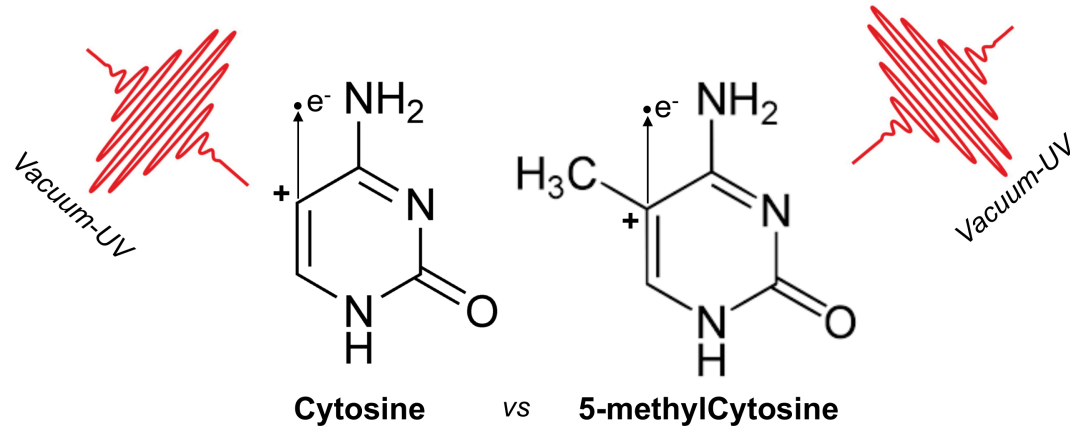

Photoionisation pathways in DNA nucleobases Cytosine and 5-methyl-Cytosine are computed at the XMS-CASPT2 level are compared to show that 5-methylation hampers the direct $D_{3} \rightarrow D_{1} \rightarrow D_{0}$ decay observed in Cytosine.

\section{References}

[1] W. J. Schreier, P. Gilch, W. Zinth, Annual Review of Physical Chemistry 2015, 66, 497-519, PMID: 25664840.

[2] F. P. Noonan, M. R. Zaidi, A. Wolnicka-Glubisz, M. R. Anver, J. Bahn, A. Wielgus, J. Cadet, T. Douki, S. Mouret, M. A. Tucker, A. Popratiloff, G. Merlino, E. C. De Fabo, Nature Communications 2012, 3, 884.

[3] C. E. Crespo-Hernández, B. Cohen, P. M. Hare, B. Kohler, Chem. Rev. 2004, 104, 1977-2020.

[4] R. Improta, F. Santoro, L. Blancafort, Chemical Reviews 2016, 116, 35403593, PMID: 26928320.

[5] A. Giussani, J. Segarra-Martí, D. Roca-Sanjuán, M. Merchán, Top. Curr. Chem. 2015, 355, 57-97.

[6] A. A. Beckstead, Y. Zhang, M. S. de Vries, B. Kohler, Phys. Chem. Chem. Phys. 2016, 18, 24228-24238. 
[7] D. B. Bucher, B. M. Pilles, T. Carell, W. Zinth, Proceedings of the National Academy of Sciences 2014, 111, 4369-4374.

[8] S. Mouret, C. Baudouin, M. Charveron, A. Favier, J. Cadet, T. Douki, Proceedings of the National Academy of Sciences 2006, 103, 13765-13770.

[9] T. Takaya, C. Su, K. de La Harpe, C. E. Crespo-Hernández, B. Kohler, Proceedings of the National Academy of Sciences 2008, 105, 10285-10290.

[10] J. Chen, Y. Zhang, B. Kohler, Top. Curr. Chem. 2015, 356, 39-87.

[11] C. T. Middleton, K. de La Harpe, C. Su, Y. K. Law, C. E. CrespoHernández, B. Kohler, Annual Review of Physical Chemistry 2009, 60, 217-239, PMID: 19012538.

[12] J. Cadet, S. Mouret, J.-L. Ravanat, T. Douki, Photochemistry and Photobiology 2012, 88, 1048-1065.

[13] A. Banyasz, T. Ketola, L. Martínez-Fernández, R. Improta, D. Markovitsi, Faraday Discuss. 2018, 207, 181-197.

[14] S. Lehnert, Biomolecular Action of Ionizing Radiation, Taylor and Francis, 2008.

[15] S. Xu, D. Guo, X. Ma, X. Zhu, W. Feng, S. Yan, D. Zhao, Y. Gao, S. Zhang, X. Ren, Y. Zhao, Z. Xu, A. Dorn, L. S. Cederbaum, N. V. Kryzhevoi, Angewandte Chemie International Edition 2018.

[16] P. O'Neill in Radiation Chemistry, Vol. 87 of Studies in Physical and Theoretical Chemistry, C. D. Jonah, B. M. Rao (Eds.), Elsevier, 2001, pp. 585 -622 .

[17] T. J. A. Wolf, F. Holzmeier, I. Wagner, N. Berrah, C. Bostedt, J. Bozek, P. Bucksbaum, R. Coffee, J. Cryan, J. Farrell, R. Feifel, T. J. Martinez, B. McFarland, M. Mucke, S. Nandi, F. Tarantelli, I. Fischer, M. Gühr, Applied Sciences 2017, 7, 681.

[18] S. L. Horton, Y. Liu, P. Chakraborty, S. Matsika, T. Weinacht, The Journal of Chemical Physics 2017, 146, 064306.

[19] M. Vacher, F. E. A. Albertani, A. J. Jenkins, I. Polyak, M. J. Bearpark, M. A. Robb, Faraday Discuss. 2016, 194, 95-115.

[20] M. Vacher, M. J. Bearpark, M. A. Robb, J. a. P. Malhado, Phys. Rev. Lett. 2017, 118, 083001.

[21] J. Segarra-Martí, T. Tran, M. J. Bearpark, Phys. Chem. Chem. Phys. 2019, DOI:10.1039/C8CP07189F.

[22] A. R. Katritzky, A. J. Waring, J. Chem. Soc. 1963, 3046-3051. 
[23] M. Lesslie, J. T. Lawler, A. Dang, J. A. Korn, D. Bím, V. Steinmetz, P. Maître, F. Turecek, V. Ryzhov, ChemPhysChem 2017, 18, 1293.

[24] M. Assmann, H. Köppel, S. Matsika, The Journal of Physical Chemistry A 2015, 119, 866-875, PMID: 25564985.

[25] M. Assmann, T. Weinacht, S. Matsika, The Journal of Chemical Physics 2016, 144, 034301.

[26] S. L. Horton, Y. Liu, P. Chakraborty, P. Marquetand, T. Rozgonyi, S. Matsika, T. Weinacht, Phys. Rev. A 2018, 98, 053416.

[27] S. Matsika, Chemical Physics 2008, 349, 356 - 362, Electron Correlation and Molecular Dynamics for Excited States and Photochemistry.

[28] M. Spanner, S. Patchkovskii, C. Zhou, S. Matsika, M. Kotur, T. C. Weinacht, Phys. Rev. A 2012, 86, 053406.

[29] M. Kotur, T. C. Weinacht, C. Zhou, K. A. Kistler, S. Matsika, The Journal of Chemical Physics 2011, 134, 184309.

[30] M. Kotur, T. Weinacht, C. Zhou, S. Matsika, IEEE Journal of Selected Topics in Quantum Electronics 2012, 18, 187-194.

[31] M. J. Bearpark, M. A. Robb, N. Yamamoto, Spectrochimica Acta Part A: Molecular and Biomolecular Spectroscopy 1999, 55, 639-646.

[32] K. F. Hall, M. Boggio-Pasqua, M. J. Bearpark, M. A. Robb, The Journal of Physical Chemistry A 2006, 110, 13591-13599, PMID: 17165887.

[33] A. M. Tokmachev, M. Boggio-Pasqua, M. J. Bearpark, M. A. Robb, The Journal of Physical Chemistry A 2008, 112, 10881-10886, PMID: 18831544 .

[34] A. M. Tokmachev, M. Boggio-Pasqua, D. Mendive-Tapia, M. J. Bearpark, M. A. Robb, The Journal of Chemical Physics 2010, 132, 044306.

[35] D. Dougherty, K. Wittel, J. Meeks, S. P. McGlynn, Journal of the American Chemical Society 1976, 98, 3815-3820.

[36] K. D. Fulfer, D. Hardy, A. A. Aguilar, E. D. Poliakoff, The Journal of Chemical Physics 2015, 142, 224310.

[37] V. Orlov, A. Smirnov, Y. Varshavsky, Tetrahedron Letters 1976, 17, 4377 -4378 .

[38] G. Lauer, W. Schäfer, A. Schweig, Tetrahedron Letters 1975, 16, 3939 3942.

[39] S. Urano, X. Yang, P. R. LeBreton, Journal of Molecular Structure 1989, 214, $315-328$. 
[40] D. Roca-Sanjuán, M. Rubio, M. Merchán, L. Serrano-Andrés, The Journal of Chemical Physics 2006, 125, 084302.

[41] P. Slavíček, B. Winter, M. Faubel, S. E. Bradforth, P. Jungwirth, Journal of the American Chemical Society 2009, 131, 6460-6467, PMID: 19374336.

[42] D. M. Close, The Journal of Physical Chemistry A 2004, 108, 10376-10379.

[43] K. de La Harpe, F. R. Kohl, Y. Zhang, B. Kohler, The Journal of Physical Chemistry A 2018, 122, 2437-2444, PMID: 29425461.

[44] F. Aquilante, J. Autschbach, R. K. Carlson, L. F. Chibotaru, M. G. Delcey, L. De Vico, I. Fdez. Galván, N. Ferré, L. M. Frutos, L. Gagliardi, M. Garavelli, A. Giussani, C. E. Hoyer, G. Li Manni, H. Lischka, D. Ma, P. Å. Malmqvist, T. Müller, A. Nenov, M. Olivucci, T. B. Pedersen, D. Peng, F. Plasser, B. Pritchard, M. Reiher, I. Rivalta, I. Schapiro, J. SegarraMartí, M. Stenrup, D. G. Truhlar, L. Ungur, A. Valentini, S. Vancoillie, V. Veryazov, V. P. Vysotskiy, O. Weingart, F. Zapata, R. Lindh, Journal of Computational Chemistry 2016, 37, 506-541.

[45] P.-O. Widmark, P.-Å. Malmqvist, B. O. Roos, Theor. Chim. Acta 1990, $77,291$.

[46] P.-O. Widmark, B. J. Persson, B. O. Roos, Theor. Chim. Acta 1991, 79, 419.

[47] K. Andersson, P. A. Malmqvist, B. O. Roos, A. J. Sadlej, K. Wolinski, J. Phys. Chem. 1990, 94, 5483-5488.

[48] K. Andersson, P.-Å. Malmqvist, B. O. Roos, J. Chem. Phys. 1992, 96, $1218-1226$.

[49] D. Roca-Sanjuán, F. Aquilante, R. Lindh, WIRES Comput. Mol. Sci. 2012, 2, 585-603.

[50] N. Forsberg, P.-Å. Malmqvist, Chem. Phys. Lett. 1997, 274, 196 - 204.

[51] G. Ghigo, B. O. Roos, P.-Å. Malmqvist, Chem. Phys. Lett. 2004, 396, 142 -149 .

[52] J. Finley, P.-Å. Malmqvist, B. O. Roos, L. Serrano-Andrés, Chem. Phys. Lett. 1998, 288, $299-306$.

[53] A. A. Granovsky, J. Chem. Phys. 2011, 134, 214113.

[54] T. Shiozaki, C. Woywod, H.-J. Werner, Phys. Chem. Chem. Phys. 2013, $15,262-269$.

[55] S. Sen, I. Schapiro, Molecular Physics 2018, 116, 2571-2582.

[56] F. Aquilante, R. Lindh, T. Bondo Pedersen, J. Chem. Phys. 2007, 127, 114107. 
[57] F. Aquilante, T. B. Pedersen, R. Lindh, J. Chem. Phys. 2007, 126, 194106.

[58] F. Aquilante, T. B. Pedersen, R. Lindh, B. O. Roos, A. Sánchez de Merás, H. Koch, J. Chem. Phys. 2008, 129, 024113.

[59] F. Aquilante, P.-Å. Malmqvist, T. B. Pedersen, A. Ghosh, B. O. Roos, J. Chem. Theory Comput. 2008, 4, 694-702.

[60] M. G. Delcey, L. Freitag, T. B. Pedersen, F. Aquilante, R. Lindh, L. González, J. Chem. Phys. 2014, 140, 174103.

[61] M. G. Delcey, T. B. Pedersen, F. Aquilante, R. Lindh, J. Chem. Phys. 2015, 143, 044110 .

[62] I. Fdez. Galván, M. G. Delcey, T. B. Pedersen, F. Aquilante, R. Lindh, Journal of Chemical Theory and Computation 2016, 12, 3636-3653, PMID: 27327873 .

[63] A. Bernhardsson, R. Lindh, J. Olsen, M. Fulscher, Molecular Physics 1999, 96, 617-628.

[64] J. Segarra-Martí, M. Garavelli, F. Aquilante, J. Chem. Theory Comput. 2015, 11, 3772-3784.

[65] J. Segarra-Martí, A. Francés-Monerris, D. Roca-Sanjuán, M. Merchán, Molecules 2016, 21, 1666.

[66] A. J. Pepino, J. Segarra-Martí, A. Nenov, R. Improta, M. Garavelli, The Journal of Physical Chemistry Letters 2017, 8, 1777-1783, PMID: 28346789 .

[67] A. J. Pepino, J. Segarra-Martí, A. Nenov, I. Rivalta, R. Improta, M. Garavelli, Phys. Chem. Chem. Phys. 2018, 20, 6877-6890.

[68] M. J. Bearpark, M. A. Robb, H. B. Schlegel, Chemical Physics Letters 1994, 223, $269-274$.

[69] T. Shiozaki, W. Győrffy, P. Celani, H.-J. Werner, The Journal of Chemical Physics 2011, 135, 081106.

[70] M. K. MacLeod, T. Shiozaki, The Journal of Chemical Physics 2015, 142, 051103.

[71] B. Vlaisavljevich, T. Shiozaki, Journal of Chemical Theory and Computation 2016, 12, 3781-3787, PMID: 27388038.

[72] J. W. Park, T. Shiozaki, Journal of Chemical Theory and Computation 2017, 13, 2561-2570, PMID: 28471661.

[73] T. Shiozaki, Wiley Interdisciplinary Reviews: Computational Molecular Science 2018, 8, e1331. 
[74] A. Giussani, J. Segarra-Martí, A. Nenov, I. Rivalta, A. Tolomelli, S. Mukamel, M. Garavelli, Theoretical Chemistry Accounts 2016, 135, 121.

[75] A. Nenov, A. Giussani, J. Segarra-Martí, V. K. Jaiswal, I. Rivalta, G. Cerullo, S. Mukamel, M. Garavelli, The Journal of Chemical Physics 2015, 142, 212443.

[76] J. Segarra-Martí, A. J. Pepino, A. Nenov, S. Mukamel, M. Garavelli, I. Rivalta, Theoretical Chemistry Accounts 2018, 137, 47.

[77] Q. Li, A. Giussani, J. Segarra-Martí, A. Nenov, I. Rivalta, A. A. Voityuk, S. Mukamel, D. Roca-Sanjuán, M. Garavelli, L. Blancafort, Chemistry - A European Journal 2016, 22, 7497-7507.

[78] J. Segarra-Martí, S. Mukamel, M. Garavelli, A. Nenov, I. Rivalta, Top. Curr. Chem. 2018, 376, 24.

[79] A. Nenov, A. Giussani, B. P. Fingerhut, I. Rivalta, E. Dumont, S. Mukamel, M. Garavelli, Phys. Chem. Chem. Phys. 2015, 17, 30925-30936.

[80] A. Nenov, R. Borrego-Varillas, A. Oriana, L. Ganzer, F. Segatta, I. Conti, J. Segarra-Marti, J. Omachi, M. Dapor, S. Taioli, C. Manzoni, S. Mukamel, G. Cerullo, M. Garavelli, The Journal of Physical Chemistry Letters 2018, 9, 1534-1541, PMID: 29504764.

[81] M. Richter, B. P. Fingerhut, Journal of Chemical Theory and Computation 2016, 12, 3284-3294, PMID: 27248511.

[82] M. Kowalewski, B. P. Fingerhut, K. E. Dorfman, K. Bennett, S. Mukamel, Chemical Reviews 2017, 117, 12165-12226, PMID: 28949133.

[83] P.-Å. Malmqvist, B. O. Roos, Chem. Phys. Lett. 1989, 155, 189-194.

[84] J. Segarra-Martí, E. Zvereva, M. Marazzi, J. Brazard, E. Dumont, X. Assfeld, S. Haacke, M. Garavelli, A. Monari, J. Léonard, I. Rivalta, Journal of Chemical Theory and Computation 2018, 14, 2570-2585, PMID: 29614854.

[85] L. Martínez-Fernández, A. J. Pepino, J. Segarra-Martí, J. Jovaišaitè, I. Vaya, A. Nenov, D. Markovitsi, T. Gustavsson, A. Banyasz, M. Garavelli, R. Improta, Journal of the American Chemical Society 2017, 139, 7780-7791, PMID: 28513173.

[86] A.-R. Allouche, Journal of Computational Chemistry 2011, 32, 174-182.

[87] G. Schaftenaar, J. Noordik, Journal of Computer-Aided Molecular Design 2000, 14, 123-134. 